\title{
Nonlinear Filter of Stochastic Approximation Type with Randomly Varying Truncations and its Acceleration*
}

\author{
Akihiko Yonemochi ${ }^{\dagger}$, Toshiharu Hatanaka ${ }^{\dagger}$, Katsuji Uosaki ${ }^{\dagger}$, and Han-Fu Chen ${ }^{\ddagger}$ \\ $\dagger$ Department of Information and Knowledge Engineering, \\ Tottori University, Tottori 680-8552, Japan \\ E-mail \{yonemoti, hatanaka, uosaki\}@ike.tottori-u.ac.jp \\ $\ddagger$ Institute of Systems Science, Chinese Academy of Science \\ Beijing 100080, P. R. China \\ E-mail hfchen@iss03.iss.ac.cn
}

\begin{abstract}
State estimation of dynamical systems is one of the most important problems in control systems engineering. This paper deals with a stochastic approximation type nonlinear filter for state estimation of nonlinear stochastic dynamical systems. Though sufficient conditions for the convergence of this nonlinear filter has been provided, they are still restrictive. We relax the restriction on the observation systems by introducing the idea of randomly varying truncations. some modifications on the algorithm is also provided to accelerate the convergence.
\end{abstract}

Keywords. Nonlinear filter; Stochastic approximation; Randomly varying truncation; Convergence; Acceleration

\section{Introduction}

State estimation of stochastic dynamical systems is one of the most important problems in control systems engineering [2],[7]. For linear dynamical systems under linear observation system with Gaussian noise, the problem is completely solved by Kalman filter [9],[12]

${ }^{*}$ This work was supported in part by the Grant-in-Aid for Scientific Research from the Japan Society for the Promotion of Science (10045043 and 10650432). under mean squared error criterion. Here, only the consideration about the convergence of the first and the second orders is sufficient. However, in case of nonlinear systems, it has not been solved yet since a posterior probability distribution, which plays a key role in Kalman filter derivation, is hardly evaluated and higher order moment convergence is not assured. Several approximations have been proposed [8], [1], but the convergence of the estimate depends on case.

Stochastic approximation type nonlinear filter, originated by Pearson [11] and then developed by Wolverton [16] is an approach to solve it . The sufficient conditions, under which this filter assures the estimation error $x_{n}-\hat{x}_{n}$ converges to zero with probability one and also in the mean square were discussed by Fujita and Fukao[6], Yoshioka et al.[15], Uosaki[13] and so on. This filter has a simple structure and is applicable to a wide class of nonlinear systems and noise distributions. However, the conditions in the past works on the nonlinear observation systems are still rather restrictive to apply. This paper is concerned with relaxing the restrictions on nonlinear observation system for this stochastic approximation type nonlinear filter. Moreover, some modifications on the algorithm is also provided to accelerate the convergence, and numerical examples illustrate the usefulness of the filter and its modifications. 


\section{Nonlinear Filter of Stochastic Approximation Type}

Consider a scalar nonlinear dynamical system described by

$$
x_{n+1}=g_{n}\left(x_{n}\right)+v_{n}, \quad n=1,2, \cdots
$$

with the observation system

$$
y_{n}=f_{n}\left(x_{n}\right)+w_{n}, \quad n=1,2, \cdots
$$

where $x_{n}, y_{n}, v_{n}$ and $w_{n}$ are state variables, observations, unknown deterministic system disturbances and observation noises, respectively, and $f_{n}$ and $g_{n}$ are nonlinear known functions. The nonlinear filter of stochastic approximation type is given by

$$
\begin{gathered}
\hat{x}_{n+1}=g_{n}\left(\hat{x}_{n}\right)+a_{n}\left(y_{n+1}-f_{n+1}\left(g_{n}\left(\hat{x}_{n}\right)\right)\right) \\
n=1,2, \cdots
\end{gathered}
$$

$\hat{x}_{1}$ : arbitrary random number such that

$$
E\left(\hat{x}_{1}^{2}\right)<\infty
$$

The sufficient conditions on the convergence by this filter were discussed by Wolverton[16], Fujita and Fukao[6], [13], et al. For example, if we assume the following Conditions,

A0. The functions $f_{n}(x)$ and $g_{n}(x)$ have continuous first derivatives for all $x$.

A1. For any $x, y$, there exist constants $C_{1}$ and $C_{2}$ such that

$$
\begin{aligned}
\left|f_{n}(x)-f_{n}(y)\right| \leq C_{1}|x-y|+C_{2} \\
\\
\text { for } \forall n=1,2, \cdots
\end{aligned}
$$

A2. For any $x, y$,

$$
\left(f_{n}(x)-f_{n}(y)\right)(x-y)>0 \text { for } \forall n=1,2, \cdots
$$

A3. Define

$$
d_{n}(x, y)=g_{n}(x)-g_{n}(y)-(x-y)
$$

then, there exists a positive number sequence $\left\{\gamma_{n}\right\}$ such that for $n=1,2, \cdots$,

$$
\begin{gathered}
\left|d_{n}(x, y)\right| \leq \gamma_{n}|x-y| \quad \text { for } \forall x, y \\
\gamma_{n}=o\left(a_{n}\right), \quad \sum_{n=1}^{\infty} \gamma_{n}<\infty
\end{gathered}
$$

A4. $\quad a_{n}>0, \quad \sum_{n=1}^{\infty} a_{n}=\infty, \quad \sum_{n=1}^{\infty} a_{n}^{2}<\infty$

A5. $\left|v_{n}\right|=o\left(a_{n}\right), \quad \sum_{n=1}^{\infty}\left|v_{n}\right|<\infty$

A6. For the observation noise sequence $\left\{w_{n}\right\}$,

$$
E\left[w_{n}\right]=0, \quad E\left[w_{n} w_{m}\right]=\sigma^{2} \delta_{m n}
$$

where $\delta_{m n}$ is Kronecker's delta.

we can prove the convergence of the estimation error to zero,

$$
\begin{aligned}
& \operatorname{Pr}\left\{\lim _{n \rightarrow \infty}\left|x_{n}-\hat{x}_{n}\right|=0\right\}=1 \\
& \lim _{n \rightarrow \infty} E\left[\left(x_{n}-\hat{x}_{n}\right)^{2}\right]=0
\end{aligned}
$$

Though the filter has a simple structure and is applicable to a wide class of nonlinear systems and noise distributions, the restrictions on the nonlinear observation systems (usually pseudo-linear as in Condition A1), are still rather strong to apply. In the following, the restrictions on nonlinear observation system will be relaxed by introducing an idea of randomly varying truncations [4].

\section{Nonlinear Filter with Ran- domly Varying Truncations}

Let us take $\hat{x}_{1}$ arbitrary as the estimate for $x_{1}$ and define a rough estimate for $x_{n+1}$ by

$$
\begin{aligned}
& h_{1}\left(\hat{x}_{1}\right)=g_{1}\left(\hat{x}_{1}\right) \\
& h_{n+1}\left(\hat{x}_{1}\right)=g_{n+1}\left(h_{n}\left(\hat{x}_{1}\right)\right) \text { for } n=1,2, \cdots
\end{aligned}
$$

In the Theorem below, some conditions will be imposed on $\left\{g_{n}(\cdot)\right\}$ and $\left\{w_{n}\right\}$ so that

$$
\left|x_{n+1}-h_{n}\left(\hat{x}_{1}\right)\right|<\eta<\infty \text { for } \forall n=1,2, \cdots
$$

where $\eta$ is an unknown constant. Thus, it can be seen that $h_{n}\left(\hat{x}_{1}\right)$ may serve as a reference for $x_{n+1}$ in the sense that if $x_{n+1}-h_{n}\left(\hat{x}_{1}\right)$ diverges to infinity then some measure on $x_{n+1}$ should be taken to prevent $x_{n+1}-h_{n}\left(\hat{x}_{1}\right)$ from going to infinity, i.e., the algorithm should be truncated. When the constant $\eta$ is known, a 
truncation with fixed bound can be used. However, expanding truncation bounds have to be used since it is unknown. This is the idea of the following algorithm. Introduce a sequence of increasing real numbers $\left\{K_{j}\right\}$ satisfying

$$
K_{j}>0, \quad K_{j+1}>K_{j}, \quad \lim _{j \rightarrow \infty} K_{j}=\infty
$$

Let $\left\{\hat{x}_{n}\right\}$ be recursively defined by the following algorithm.

$$
\begin{aligned}
\tilde{x}_{n+1} & =g_{n}\left(\hat{x}_{n}\right)+a_{n}\left(y_{n+1}-f_{n+1}\left(g_{n}\left(\hat{x}_{n}\right)\right)\right) \\
\hat{x}_{n+1} & =\tilde{x}_{n+1} I_{\left[\left|\tilde{x}_{n+1}-h_{n}\left(\hat{x}_{1}\right)\right| \leq K_{\sigma_{n}}\right]} \\
& +h_{n}\left(\hat{x}_{1}\right) I_{\left.\left[\mid \tilde{x}_{n+1}-h_{n}\left(\hat{x}_{1}\right)\right) \mid>K_{\sigma_{n}}\right]} \\
\sigma_{n} & =\sum_{i=1}^{n-1} I_{\left.\left[\mid \tilde{x}_{n+1}-h_{n}\left(\hat{x}_{1}\right)\right) \mid>K_{\sigma_{i}}\right]}
\end{aligned}
$$

Here, $\sigma_{n}$ is the number of truncations occurred until time $n$. The algorithm implies that at time $n+1$, the estimate $g_{n}\left(\hat{x}_{n}\right)+a_{n}\left(y_{n+1}-f_{n+1}\left(g_{n}\left(\hat{x}_{n}\right)\right)\right)$ given by the conventional stochastic approximation type nonlinear filter (3), is compared with a rough estimate for $x_{n+1}$, $h_{n}\left(\hat{x}_{1}\right)$. If the difference is less than the truncation bound $K_{\sigma_{n}}$, then the estimate $\tilde{x}_{n+1}$ is accepted as the estimate $\hat{x}_{n+1}$. Otherwise, $h_{n}\left(\hat{x}_{1}\right)$ is employed for the estimate $\hat{x}_{n+1}$.

The convergence theorem for the nonlinear filter with randomly varying truncations is given as follows.

Theorem. In additions to the Conditions A0, A3 and A5, we assume the following Conditions

A1'. For any $c>0$, there is a constant $\alpha(c)$ possibly depending on $c$ such that

$$
\begin{aligned}
& \qquad\left|f_{n}(x)-f_{n}(y)\right|<\alpha(c) \\
& \text { for } \forall x, y \text { with }|x-y| \leq c, \forall n=1,2, \cdots
\end{aligned}
$$

A2'. There is a continuously differentiable function $v(x)$ such that

$$
\begin{gathered}
v(x) \neq 0 \text { for } \forall x \neq 0, \quad v(0)=0 \\
\inf _{n} \inf _{r_{1} \leq|x-y| \leq r_{2}}\left(f_{n}(x)-f_{n}(y)\right) v^{\prime}(x-y)>a \\
\text { for any } 0<r_{1}<r_{2}<\infty
\end{gathered}
$$

where $a$ is a positive constant possibly depending on $r_{1}$ and $r_{2}$, and $v^{\prime}(x)$ denotes the derivative of $v(x)$. It is also required that for $\eta$ in eq.(7), there exists a constant $r>\eta$ such that

$$
\sup _{|y| \leq \eta} v(y)<\sup _{|x|=r} v(x)
$$

A4'. $\quad a_{n}>0, \quad \lim _{n \rightarrow \infty} a_{n}=0, \quad \sum_{n=1}^{\infty} a_{n}=\infty$

A6'. For any convergent subsequence $\left\{\hat{x}_{n_{i}}-x_{n_{i}}\right\}$ the observation noise satisfies

$$
\begin{gathered}
\lim _{T \rightarrow 0} \limsup _{i \rightarrow \infty} \frac{1}{T}\left|\sum_{\substack{j=n_{i} \\
\text { for } \forall t \in[0, T]}}^{m\left(n_{i}, t\right)} a_{j} w_{j+1}\right|=0 \\
\text {. }
\end{gathered}
$$

$$
\text { with } m(n, T)=\max \left\{m \mid \sum_{i=n}^{m} a_{i} \leq T\right\}
$$

then, the estimation error $x_{n}-\hat{x}_{n}$ converges to zero as $n \rightarrow \infty$ as in 5 .

Remark. Condition A1' implies the local bounded property of the function $f_{n}(x)$ with uniform upper bound with respect to $n$. Condition A2' is about existence of a Lyapunov function. Noise conditions are given in Condition A6'. This condition is necessary for $x_{n}-\hat{x}_{n} \rightarrow 0$ as $n \rightarrow \infty$ if the Conditions in the Theorem hold.

This theorem can be proved based on the following lemmas that show the bounded property of the estimation error and finite property of the number of truncations. (The proofs of the lemmas are in [14].)

Lemma 1. Under Conditions A3 and A5, the sequence $\left\{x_{n+1}-h_{n}\left(\hat{x}_{1}\right)\right\}$ is bounded for any $\hat{x}_{1}$.

Let

$$
\Delta_{n}=x_{n}-\hat{x}_{n}
$$

Lemma 2. Assume Conditions A0, A1', A3', A4', A5 and A6'. Let $\left\{\Delta_{n_{i}}\right\}$ be a convergent subsequence such that $\Delta_{n_{i}} \rightarrow \bar{\Delta}$ as $i \rightarrow \infty$. Then, there are sufficiently small $T>0$ and a sufficiently large integer $i_{0}$ such that for $i \geq i_{0}$

$$
\begin{aligned}
& \hat{x}_{m+1}=\tilde{x}_{m+1} \\
& =g_{m}\left(\hat{x}_{m}\right)+a_{m}\left(y_{m+1}-f_{m+1}\left(g_{m}\left(\hat{x}_{m}\right)\right)\right) \\
& \left|\Delta_{m}-\Delta_{n_{i}}\right| \leq c t
\end{aligned}
$$

for $\forall m$ satisfying $n_{i} \leq m \leq m\left(n_{i}, t\right), \forall t \in[0, T]$, where $c$ is a constant independent of $i$. 
The lemma implies that for sufficiently large $i$, starting from $n_{i}$, the filter has no truncations and the estimation error at time $m$ is close to that at time $n_{i}$ for any $m \in\left[n_{i}, m\left(n_{i}, T\right)\right]$.

Lemma 3. Under the Conditions in the Theorem, the number of truncations in eq.(9) is finite and $\left\{\Delta_{n}\right\}$ is bounded.

This lemma implies the bounded property of $\left\{\Delta_{n}\right\}$.

Now we are at the position to prove the Theorem.

Proof of Theorem. First, it is shown that $v\left(\Delta_{n}\right)$ converges. Assume the converse:

$$
v_{1}=\liminf _{n \rightarrow \infty} v\left(\Delta_{n}\right)<\limsup _{n \rightarrow \infty} v\left(\Delta_{n}\right)=v_{2}
$$

where $-\infty<v_{1}<v_{2}<\infty$ because $\left\{\Delta_{n}\right\}$ is bounded by Lemma 3 . It is clear that there exists an interval $\left[\delta_{1}, \delta_{2}\right]$ that does not contain zero such that $\left[\delta_{1}, \delta_{2}\right] \subset\left(v_{1}, v_{2}\right)$. Without loss of generality, assume $0<\delta_{1}<\delta_{2}$. From Condition A2', it follows that there are infinitely many sequences such that $v\left(\Delta_{m}\right) \leq \delta_{1}, v\left(\Delta_{\ell_{i}}\right) \geq \delta_{2}$ and that $v\left(\delta_{j}\right) \in\left(\delta_{1}, \delta_{2}\right)$ for $j$ satisfying $m_{i}<j<\ell_{i}, i=1,2, \cdots$. Without loss of generality, it is assumed that $\left\{\Delta_{m_{i}}\right\}$ converges, since a convergent subsequences can be always selected from $\left\{\Delta_{m_{i}}\right\}$. Let $\Delta_{m_{i}} \rightarrow \Delta^{*}$. Since $v\left(\Delta^{*}\right)=\delta_{1}>0$, there is $\xi>0$ such that $\left|\Delta^{*}\right| \geq \xi$, and by Lemma $2\left|\Delta_{j}\right|<\xi / 2$ for $\forall j$ satisfying $m_{i} \leq j \leq m\left(m_{i}, T\right)$. Completely by the same argument used in the Proof of Lemma 3 leads to a contradiction. Hence $v\left(\delta_{n}\right)$ is convergent.

Now it is shown that $\Delta_{n} \rightarrow 0$ as $n \rightarrow \infty$. Assume that the converse: there is a subsequence $\Delta_{m_{i}} \rightarrow \Delta^{*} \neq 0$. The same argument in the Proof of Lemma 3 [14] leads to

$$
v\left(\Delta_{m\left(m_{i}, T\right)}\right)-v\left(\Delta_{m_{i}}\right)>\frac{a}{2} T
$$

By tending $i \rightarrow \infty$, we have a contradictive inequality $0>a T / 2$ by convergence property of $\left\{v\left(\delta_{n}\right)\right\}$. Hence we have $\Delta_{n} \rightarrow 0$ as $n \rightarrow \infty$.

\section{Acceleration Technique}

As shown in the preceding section, the nonlinear filter proposed assures the convergence to the true state, but it is asymptotic. For small number of observation, its convergence may sometimes slow. We can see that the estimation error the residual $y_{n+1}-f_{n+1}\left(g_{n}\left(\hat{x}_{n}\right)\right)$ is same (positive or negative) when the estimation error $\hat{x}_{n}-x_{n}$ is large and it often changes the sign when the estimation error is small. Based on this observation, we reduce the gain $a_{n}$ in order to give large correction to the estimate $\hat{x}_{n}$ only when the residual $y_{n+1}-f_{n+1}\left(g_{n}\left(\hat{x}_{n}\right)\right)$ changes its sign instead of employing monotone decreasing number sequence as the gain $\left\{a_{n}\right\}$. Thus, we have the following acceleration algorithm:

$$
\begin{aligned}
\tilde{x}_{n+1} & =g_{n}\left(\hat{x}_{n}\right)+a_{\tau_{n}}\left(y_{n+1}-f_{n+1}\left(g_{n}\left(\hat{x}_{n}\right)\right)\right) \\
\hat{x}_{n+1} & =\tilde{x}_{n+1} I_{\left[\left|\tilde{x}_{n+1}-h_{n}\left(\hat{x}_{1}\right)\right| \leq K_{\sigma_{n}}\right]} \\
& +h_{n}\left(\hat{x}_{1}\right) I_{\left.\left[\mid \tilde{x}_{n+1}-h_{n}\left(\hat{x}_{1}\right)\right) \mid>K_{\sigma_{n}}\right]} \\
\sigma_{n} & =\sum_{i=1}^{n-1} I_{\left.\left[\mid \tilde{x}_{n+1}-h_{n}\left(\hat{x}_{1}\right)\right) \mid>K_{\sigma_{i}}\right]} \\
\tau_{n} & =\sum_{i=1}^{n-1} I_{\left[\left(y_{i+1}-f_{i+1}\left(g_{i}\left(\hat{x}_{i}\right)\right)\right)\left(y_{i}-f_{i}\left(g_{i-1}\left(\hat{x}_{i-1}\right)\right)\right)<0\right]} \\
a_{n} & \leq a_{n+1}
\end{aligned}
$$

The convergence of this acceleration algorithm can be proved in parallel to that of Kesten[10].

\section{$5 \quad$ Numerical Examples}

We give illustrative numerical examples.

\section{Example 1}

Consider the following dynamical system:

$$
\begin{aligned}
& x_{n+1}=g_{n}\left(x_{n}\right)=\frac{\pi}{n} \cos \left(\frac{n x_{n}}{\pi}\right) \quad n=1,2, \ldots \\
& x_{1}=1
\end{aligned}
$$

with observation system

$$
\begin{aligned}
& y_{n}=\frac{x^{3}}{100}+w_{n} \\
& w_{n} \sim N(0,25)
\end{aligned}
$$

The sample behaviors of true state and estimates defined by (3) and (9) with $\hat{x}_{1}=3, a_{n}=2 / n$ are given in Figure 1. Random varying truncations provides good tracking ability.

\section{Example 2}

Consider the following dynamical system:

$$
\begin{aligned}
& x_{n+1}=g_{n}\left(x_{n}\right)=\left(\frac{2(-1)^{n+1}}{n^{2}}+1\right) x_{n}, \quad n=1,2, \ldots \\
& x_{1}=1
\end{aligned}
$$




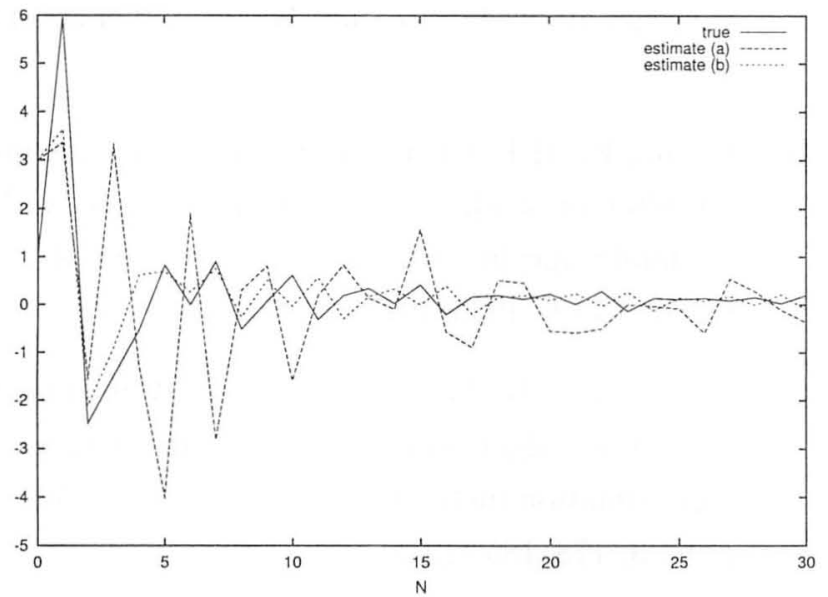

Figure 1: Sample behaviors of the state estimates and true state for Example 1. (a) estimate by conventional SA; (b) estimate by SA with truncations

with same observation system, where we consider the cases $w_{n} \sim N(0,1)$ and $w_{n} \sim N(0,0.1)$. Figure 2 gives the sample behaviors of true state and estimates given by (3),(9) and (15) with $\hat{x}_{1}=2, a_{n}=1.6 / n$ for the case of $\sigma^{2}=1$, and Figure 3 gives the sample behaviors of mean square errors. These show the usefulness of the ideas of random varying truncations and acceleration.

\section{Conclusion}

We have discussed sufficient conditions for the convergence of a nonlinear filter of stochastic approximation type for state estimation of nonlinear dynamical systems. By introducing the idea of randomly varying truncations, we can relax the restrictions on the observation systems. Moreover, some modifications on the algorithm is also provided to accelerate the convergence. Numerical examples illustrate the usefulness of the filter and its modifications.

\section{References}

[1] Special issue on application of Kalman filtering, IEEE Trans. on Automat. Contr., AC-28, No.3 (1983).

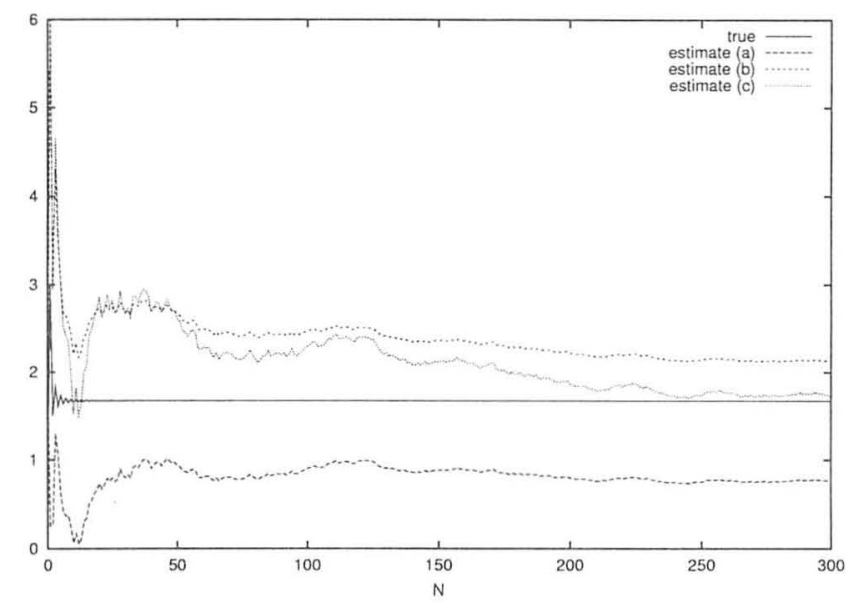

Figure 2: Sample behaviors of the state estimates and true state for Example 2 with $\sigma^{2}=1$. (a) estimate by conventional SA; (b) estimate by SA with truncations; (c) estimate by accelerated SA with truncations.

[2] Anderson, B. D. O. and J. B. Moore, Optimal Filtering, Prentice Hall, Englewood Cliffs, NJ (1968).

[3] Chen, H-F., Continuous time stochastic approximation: convergence and asymptotic efficiency, Stochastics and Stochastics Repts., 51, 111-132 (1994).

[4] Chen, H-F., Recent developments in stochastic approximation, Proc. IFAC World Congress, D, 375-380 (1996).

[5] Chen, H-F. and Y-M. Zhu, Stochastic approximation procedures with randomly varying truncations," Scientia Sinica (Series A), 29, 914-926 (1986).

[6] Fujita, S. and T. Fukao, Convergence conditions of dynamic stochastic approximation method for nonlinear stochastic discrete-time system, IEEE Trans. Auto. Contr., AC-17, 715-717 (1972).

[7] Haykin, S., Adaptive Filter Theory (2nd Ed.), Prentice Hall, Englewood Cliffs, NJ (1991).

[8] Jaswinski, A. H., Stochastic Processes and Filtering Theory, Academic Press, New York, NY (1970). 


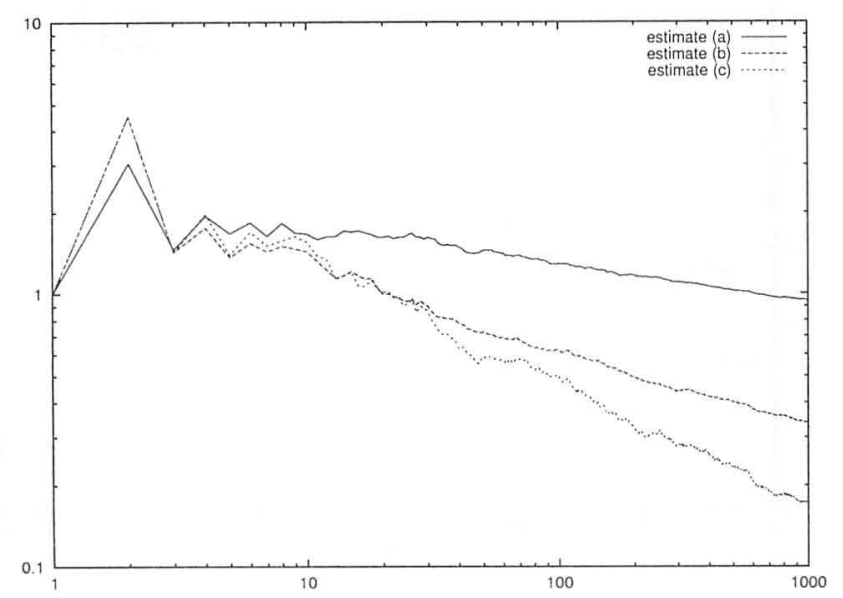

(a) $\sigma^{2}=1$

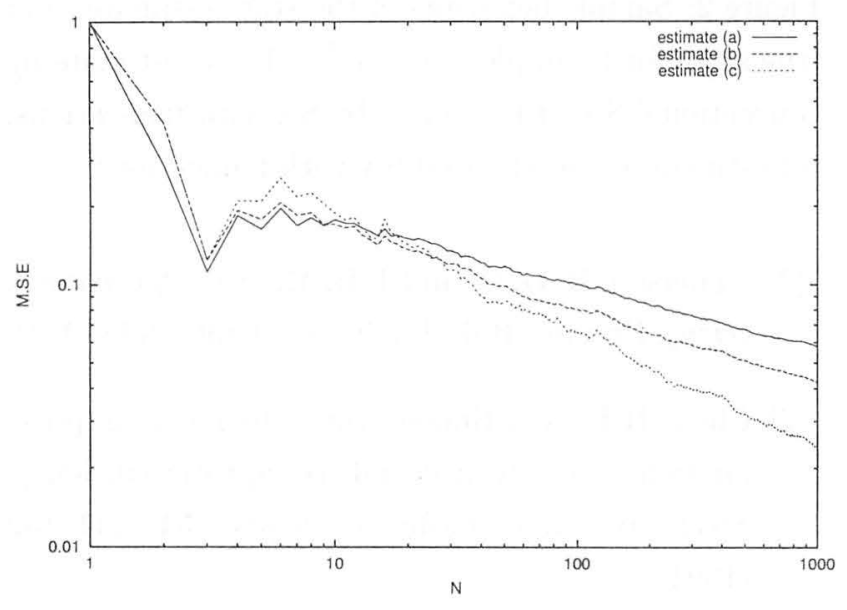

(b) $\sigma^{2}=0.1$

Figure 3: Mean square errors for Example 2

[9] Kalman, R. E., A new approach to linear filtering and prediction problems, Trans. ASME, J. Basic Engineering, 82, 35-45 (1960).

[10] Kesten, H., Accelerated stochastic approximation, Ann. Math. Statist., 29, 41-59 (1958).

[11] Pearson, J. B., A note on nonlinear filtering, IEEE Trans. Auto. Contr., AC-13, 103-105 (1968).

[12] Sorenson, H. W. (ed.), Kalman Filtering: Theory and Applications, IEEE Press, New York, NY (1985).

[13] Uosaki, K., State estimation scheme for nonlinear dynamical systems based on the stochastic approximation, Information Sciences, 9, 133-149 (1975).

[14] Uosaki, K., H-F. Chen and T. Hatanaka, Nonlinear filter of stochastic approximation type with randomly varying truncations, Proc. 3rd Asian Contr. Conf., 1923-1927 (2000).

[15] Yoshioka, Y., K. Abe and H. Takeda, A nonlinear estimation algorithm based on the stochastic approximation method, Trans. Soc. Instr. Contr. Eng., 9, 178-185 (1973)

[16] Wolverton, C. T. A note on the application of Dvoretzky's theorem to nonlinear filtering, IEEE Trans. Auto. Contr., AC-14, 117-119 (1969) 